\title{
COMPONENT-BASED DEVELOPMENT OF OBSERVATIONAL SOFTWARE FOR KASI SOLAR IMAGING SPECTROGRAPH
}

\author{
Seonghwan Choi ${ }^{1}$, Yeon-Han Kim ${ }^{1}$, Yong-Jae Moon ${ }^{1}$, Kyung-Seok Cho ${ }^{1}$, \\ Young-Deuk PARK ${ }^{1}$, BI-Ho JANG ${ }^{1}$, Su-JIN KIM ${ }^{1,2}$, and KAP-Sung KIM ${ }^{2}$ \\ ${ }^{1}$ Solar and Space Weather Research Group, Korea Astronomy and Space Science Institute, \\ Daejeon 305-348, Korea \\ E-mail: shchoi@kasi.re.kr,yhkim@kasi.re.kr,yjmoon@kasi.re.kr,kscho@kasi.re.kr, \\ ydpark@kasi.re.kr, \& bhjang@kasi.re.kr \\ 2 Department of Astronomy and Space Science, Kyunghee University, Yongin 446-701, Korea \\ E-mail:sjkim@kasi.re.kr@ kskim@khu.ac.kr \\ (Received November 15, 2005; Accepted November 25, 2005)
}

\begin{abstract}
In this paper, we have made the component-based development of observational software for KASI solar imaging spectrograph (KSIS) that is able to obtain three-dimensional imaging spectrograms by using a scanning mirror in front of the spectrograph slit. Since 2002, the KASI solar spectrograph has been successfully operated to observe solar spectra for a given slit region as well as to inspect the response functions of narrow band filters. To improve its capability, we have developed the KSIS that can perform sequential observations of solar spectra by simultaneously controlling the scanning mirror and the CCD camera via Visual $\mathrm{C}++$. Main task of this paper is to introduce the development of the component-based software for KSIS. Each component of the software is reusable on the level of executable file instead of source code because the software was developed by using CBD (componentbased development) methodology. The main advantage of such a component-based software is that key components such as image processing component and display component can be applied to other similar observational software without any modifications. Using this software, we have successfully obtained solar imaging spectra of an active region (AR 10708) including a small sunspot. Finally, we present solar $\mathrm{H} \alpha$ spectra $(6562.81 \AA)$ that were obtained at an active region and a quiet region in order to confirm the validity of the developed KSIS and its software.
\end{abstract}

Key words : solar imaging spectrograph, component — based development, observational software

\section{INTRODUCTION}

Solar spectrographic observations have been widely used as an useful diagnostic tool for examination of the physical conditions of chromospheric activity such as $\mathrm{H} \alpha$ flares. A better understanding of the solar phenomenon requires multi-line observations and twodimensional spectroscopy, which can reveal the dynamic behavior of the Sun at different locations and wavelengths. By using imaging spectrograph systems, several authors have studied chromospheric activity; for example, comparison with electric current systems (Canfield et al. 1993), velocity distribution by line asymmetry (Ding 1995), and physical parameters of a solar flare (Liu \& Ding 2001).

A spectrograph and an imaging spectrograph have different data forms. The spectrogram is 2-D spectral image, like $I=f(x, \lambda)$, where $x$ is spatial information and $\lambda$ is wavelength. The imaging spectrogram is $3-\mathrm{D}$ data, like $I=f(x, y, \lambda)$, where $x$ and $y$ is spatial image (2-D) that correspond to filtergram, and the other is wavelength. The imaging spectrogram is composed of a series of spectrogram or filtergram.
There are two ways to take an imaging spectrogram. Two ways are illustrated in Figure 1. One is a way of spatial scanning, which takes spectral data for a given slit position and then move the slit position. The other is a way of wavelength scanning, which takes a spatial image with a narrow-band filter and then change the central wavelength of the filter. In general, the spectral resolution of the former is better than that of the latter, and the spatial resolution of the former is worse than that of the latter.

There have been several studies on the development of solar imaging spectrographs in visible waveband since the early in the 1990's. (1) An instrument (THEMIS) using the multi-channel subtractive double pass (MSDP) technique has been used with the large spectrograph of the Vacuum Tower Telescope of the Teide Observatory, as a result of cooperation between the Paris Meudon Observatory and the Kiepenheuer Institute (Mein 1977, 1991; Geppatelli \& Briand 2003). (2) The Mees CCD (MCCD) instrument is an imaging spectroscopy device which uses the $25 \mathrm{~cm}$ coronagraph telescope and the $3.0 \mathrm{~m}$ Coude spectrograph at Mees Solar Observatory (MSO) on Haleakala, Maui (Penn et al. 1991). (3) An instrument using a universal bire-

Corresponding Author: S. Choi 


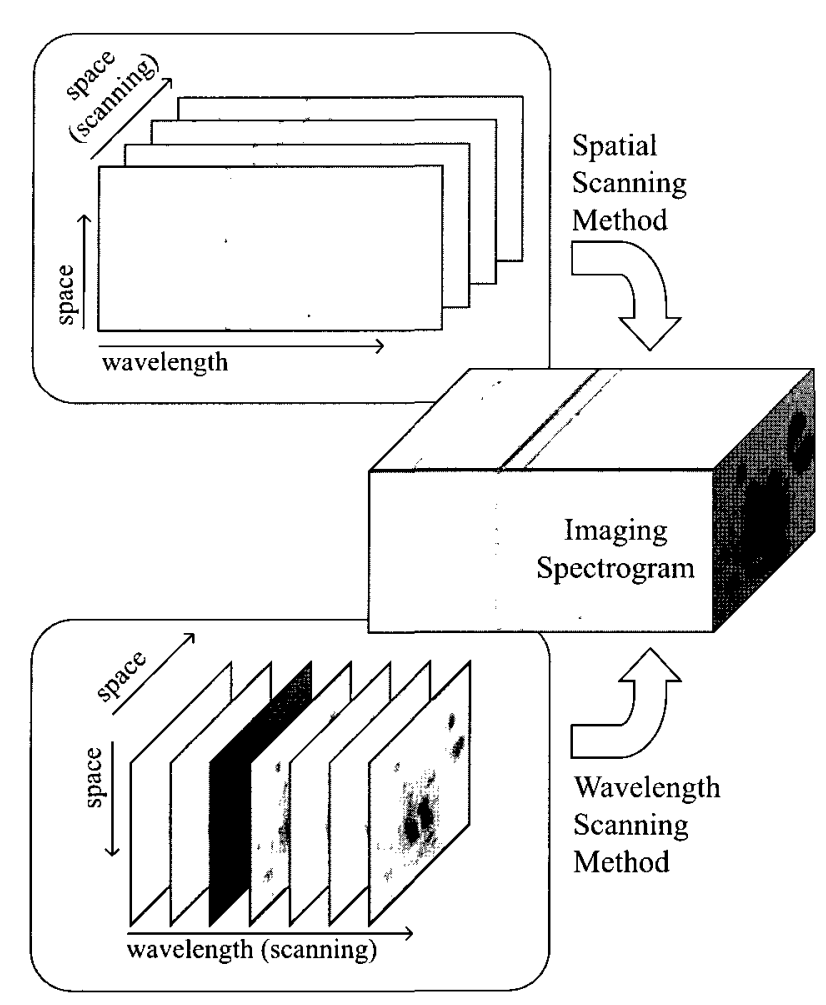

Fig. 1.- Two ways to take an imaging spectrogram

fringent filter (UBF) and a Fabry-Perot interferometer (FPI) was built and successfully tested with the German Vacuum Tower Telescope at the Observatorio del Teide/Tenerife (Bendlin et al. 1992). (4) A multichannel solar spectrograph attached to the solar tower telescope of Nanjing University, originally established in 1982, was replaced by a two-channel imaging spectrograph (Huang et al. 1995).

The scanning ways of both the MCCD and the solar tower telescope of Nanjing University are similar to that of KSIS. Table 1 shows the specifications of the above solar imaging spectrographs together with those of KSIS.

Main task of this paper is to introduce the development of an observational software for the KSIS. For this we adopt the Component Object Model (COM) technology for Microsoft Windows platform although there are two other component-based models; CORBA and JavaBeans. Windows platform is widely used, easy to use, and familiar to an operator. The reasons why we adopt the component-based development are as follows:

- It is more reusable and extensible than other programming technologies because COM is a binary standard.

- After first installation, it also allows applications and systems to be built from components supplied by different software vendors or developers.
TABLE 1.

SPECIFICATIONS OF SOLAR IMAGING SPECTROGRAPHS

\begin{tabular}{|c|c|c|c|}
\hline Parameters & MCCD & $\begin{array}{c}\text { Nanjing } \\
\text { University }\end{array}$ & KSIS \\
\hline Telescope type & Coude & Coelostat & Coelostat \\
\hline $\begin{array}{l}\text { Grating spectrum } \\
\text { order }\end{array}$ & 1st & $2 n d$ & $3 r d$ \\
\hline $\begin{array}{l}\text { Grating grooves } \\
\text { (groove } \mathrm{mm}^{-1} \text { ) }\end{array}$ & $600 / 300$ & 600 & 600 \\
\hline Focal length(mm) & 3,000 & 7,000 & 9,000 \\
\hline Aperture (mm) & - & 230 & 300 \\
\hline Diffraction angle & $\begin{array}{l}11^{\circ} 22^{\prime} \\
/ 59^{\circ} 52^{\prime}\end{array}$ & $44^{\circ} 28^{\prime}$ & $34^{\circ}$ \\
\hline $\begin{array}{l}\text { Spectral resolution } \\
(\AA)\end{array}$ & 0.014 & - & 0.2 \\
\hline $\begin{array}{l}\text { Spatial resolution } \\
\left(\operatorname{arcsec} \mathrm{mm}^{-1}\right)\end{array}$ & 0.6 & 66.55 & 20.69 \\
\hline
\end{tabular}

- Some components developed for KSIS in this paper can be used for other observational software.

This paper is organized as follows. In section II, we will introduce KSIS, mainly its hardware system. In section III, we will describe the component-based development of observational software for KSIS. In section IV, we present our test observation of an active region, AR10708. Finally, a summary and discussion of this paper will be given in section $\mathrm{V}$.

\section{KASI SOLAR IMAGING SPECTROGRA -PH}

In 2002, a solar spectroscopic system with the coelostat type was installed at Korea Astronomy and Space Science Institute (KASI). It was designed to observe solar spectra in the range from $3500 \AA$ to $7000 \AA$ with the spectral resolution of $0.2 \AA \mathrm{mm}^{-1}$. Its details were described by Park et al. (2003).

By improving the solar spectrograph, we have made the solar imaging spectrograph. For this, we have made several changes as follows. A flat mirror (M4) in front of a spectrograph slit was changed to be rotated. We call it a scanning mirror. When the scanning mirror rotates by one step, the solar image on a slit moves by $70 \mu \mathrm{m}$, which corresponds to the width size of the slit. The previous interface of the CCD camera was a LPT (Line Print Terminal) parallel port. It takes about 1 minute to take a full frame. We replaced the interface of the CCD camera with USB (Universal Serial Bus) port in order to get the high performance of transfer rates, because the solar imaging spectrograph requires a lot of image frames for a single imaging spectrogram. After changing it, it takes about 3.7 seconds to get a full single frame.

The KSIS consists of four elements; a coelostat, optical systems, a CCD camera, and software. Figure 2 shows schematic diagram of the KSIS system. The coelostat is composed of the primary mirror (PM) and 
Path of the rays from the Sun

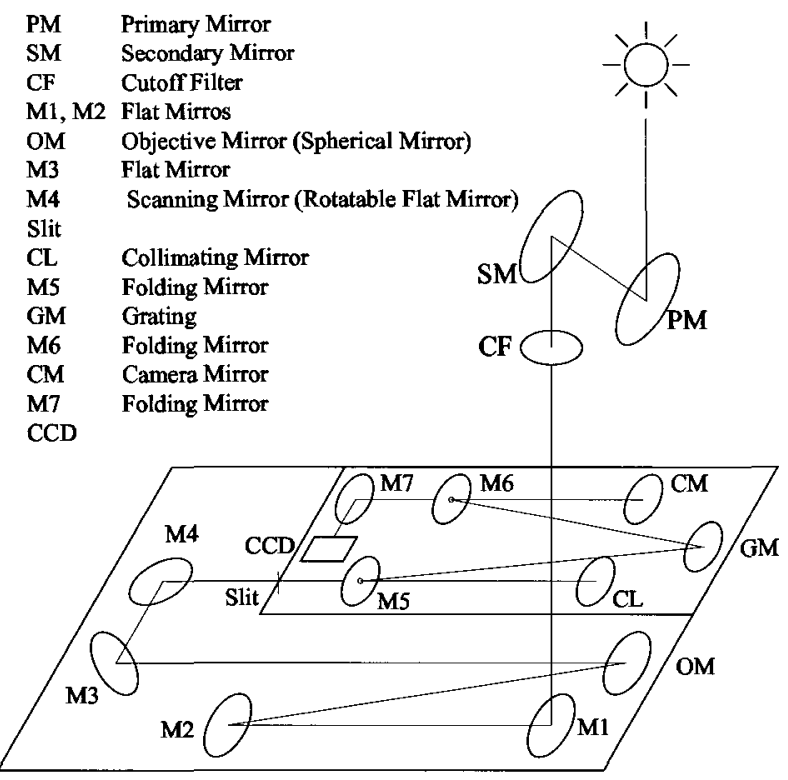

Fig. 2.- Schematic diagram of the KSIS system

the secondary mirror (SM), which is in a dome on the 2nd floor. The ray from the coelostat goes to a flat mirror in the first floor via the cutoff filter (CF). The cutoff filter only accepts the radiation covering from $3500 \AA$ to $7000 \AA$. It can prohibit the mirrors from being heated by cutting off infrared and ultraviolet rays. Then the rays are guided into a darkroom through a slit via three flat mirrors (M1, M2, M3), the objective mirror (OM), and the scanning mirror (M4). Figure 3 shows the scanning mirror. There are three folding mirrors (M5, M6, M7), the collimating mirror (CL), the grating mirror (GM), the camera mirror (CM), and the CCD camera (CCD) in the darkroom.

\section{COMPONENT-BASED DEVELOPMENT OF OBSERVATIONAL SOFTWARE}

The observational software has been developed via Visual $\mathrm{C}++$ for Windows operating system. Figure 4 shows the architecture of the observational software for the KSIS. The observational software for the KSIS consists of four components: a data acquisition component, a data display component, a data processing component, and a data archive component. By controlling the scanning mirror via a COM port and the CCD camera via a USB port, the data acquisition component can get solar imaging spectrograms. 'COM port' means RS-232C serial communication port and 'COM' means Component Object Model. The data processing component can modify the data using image processing routines in the component and save them in a FITS (Flexible Image Transport System) file. Both the data acquisition component and the data processing compo-

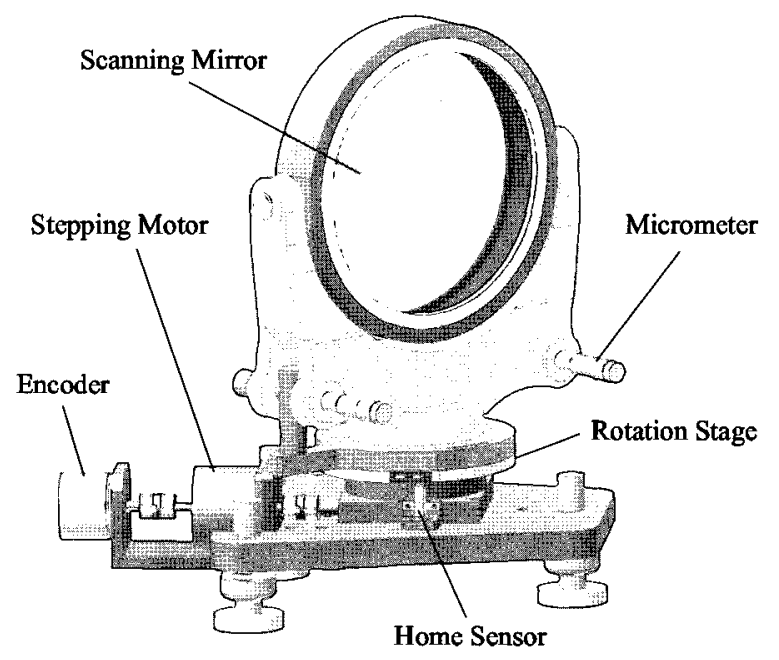

Fig. 3.- Scanning mirror

nent are the objects based on COM technology. The data display component, as a main observational application part, manipulates and displays the data from the FITS file. It can display spatial images for given wavelengths, spectral images, and spectral line profiles for given positions. The data archive component manages the observed data and display them on the internet by using Microsoft IIS (Internet Information Server). The internet services are performed via FTP (File Transfer Protocol) and HTTP (Hyper-Text Transfer Protocol).

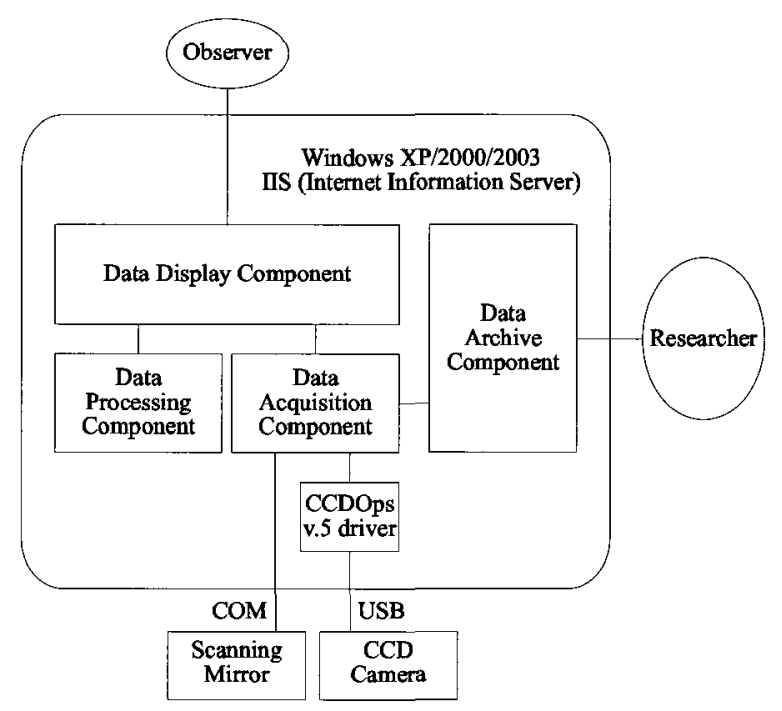

Fig. 4.- Architecture of the observational software 


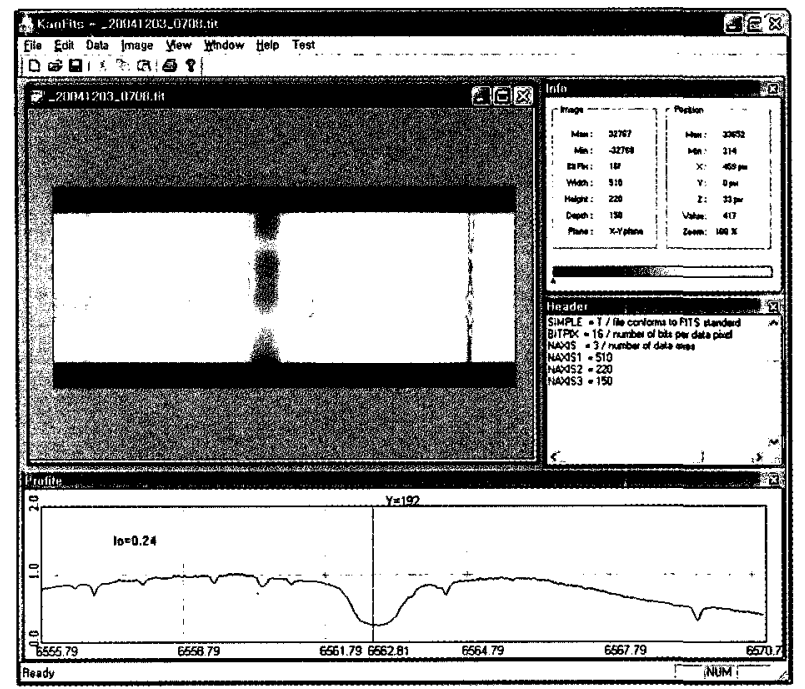

Fig. 7.- Screen shot of the data display component

Although the BITPIX of the FITS file is bigger than 8 bit, the computer can only display the intensity variation by 8 bit ( 256 levels). Thus the data display component uses the Adobe color table (.act) file. We have only to locate the Adobe color table files in "Color Tables" folder in order to use the color table.

\section{(c) Data Processing Component}

The data processing component can modify the data according to an users purpose. We can add new data processing components for the KSIS at any time. Figure 8 shows a CProcessor class and IProcessor interface as an illustration. CProcessor class has three public methods, which are GetInformation(), SetFitsFilePath(), and StartProcessor(). All data processing components must satisfy interface and class structure like this. Now we support three components, which are invert, calibration, and geometric transformation.

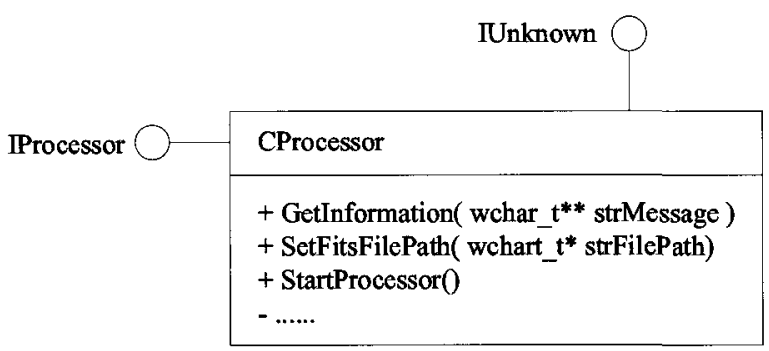

Fig. 8.- CProcessor class and IProcessor interface

\section{(d) Data Archive Component}

The observed data is distributed via the internet (FTP and HTTP) by using Microsoft Internet Information Service (IIS). Web pages are developed via JScript and based on ASP (Active Server Pages).

\section{TEST OBSERVATION}

In order to verifying the spectrum from KSIS, we used a solar atlas observed with the Fourier Transform Spectrometer at the MacMath-Pierce Solar Facility at Kitt Peak National Observatory (Kurucz et al. 1984). This series of solar spectra is taken as a reference for comparing the spectrum of the Sun. This data cover a wavelength range from $2960 \AA$ to $8000 \AA$ in steps of $0.005 \AA$. We compared observed spectra in $\mathrm{H} \alpha$ and Sodium band from a quiet region with that from the solar atlas. Figure 9 shows the solar spectrum of the KPNO's solar atlas (above) and KSIS (below). The left side images are (a) a spectral line from KPNO's atlas, (b) a spectral line from KSIS, (c) a spectral image from KSIS at $\mathrm{H} \alpha$ waveband $(6562.8 \AA)$. And the right side images are (d) a spectral line from KPNO's atlas, (e) a spectral line from KSIS, (f) a spectral image from KSIS at sodium waveband D1 $(5896 \AA)$ and D2 $(5890 \AA)$. As seen in the figures, the comparison shows that our observed spectra are well consistent with those from KPNO's Atlas.

By using the KSIS, we have observed an active region (AR 10708) on 2004 December 3. We have obtained a solar imaging spectrogram and analyzed the 3-D data by using the observational software that has developed in this paper. Figure 10 shows the spectral image and the line profiles at different positions. In the left-top spectral image, the horizontal dark region indicates a sunspot. And the relatively bright region in the $\mathrm{H} \alpha$ waveband corresponds to an active region. The images below the spectral image are spectral line profiles at the different positions indicated in the spectrogram. As seen in the second profile (b), the $\mathrm{H} \alpha$ line has a more shallow depth than others, implying that there existed chromospheric emission processes in the active region. To clarify this argument, we present the relative intensity (the right images) given by $f_{r}(\lambda)=f(\lambda)-f_{m}(\lambda)$, where $f_{m}(\lambda)$ is a mean spectrum for quiet regions as a reference. As seen in the figure, we can see clearly enhanced emissions in the line center relative to the spectrum of quite regions.

Figure 11 shows spatial images of an active region AR 10708 for several selected wavelengths, which is constructed from the imaging spectrogram. Its field of view is $196 \operatorname{arcsec} \times 102$ arcsec. In the spatial images taken at the line wings $\left(\lambda-\lambda_{0} \geq 0.9 \AA\right)$, we can see a sunspot, as seen in the continuum images. Especially in the line center regions, we can see several emission patches and absorption features in the active region. 

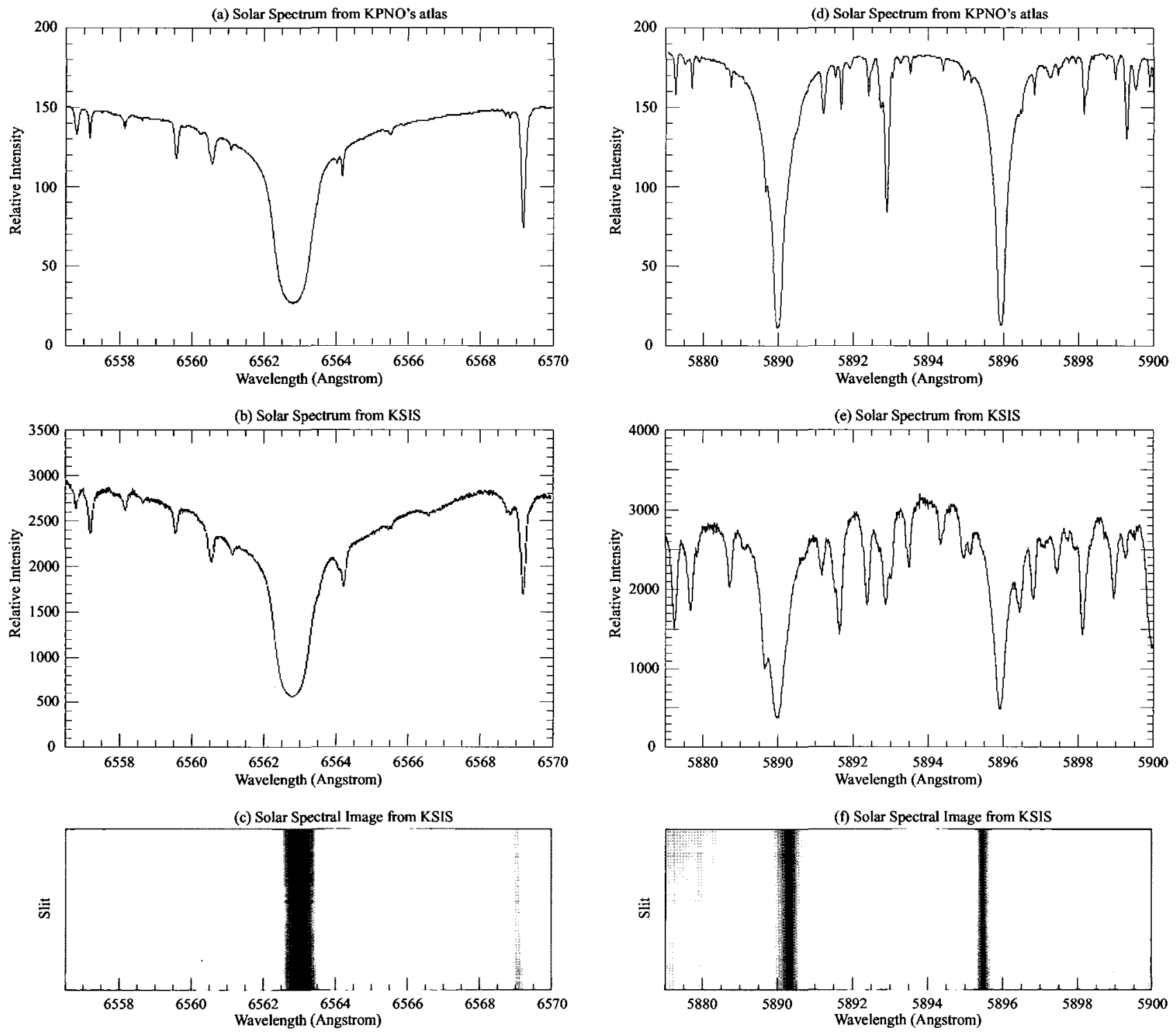

Fig. 9.- Solar spectrum from KPNO's atlas and KSIS at H $\alpha$ waveband and sodium waveband. 
(a)

(b)

(c)

(d)
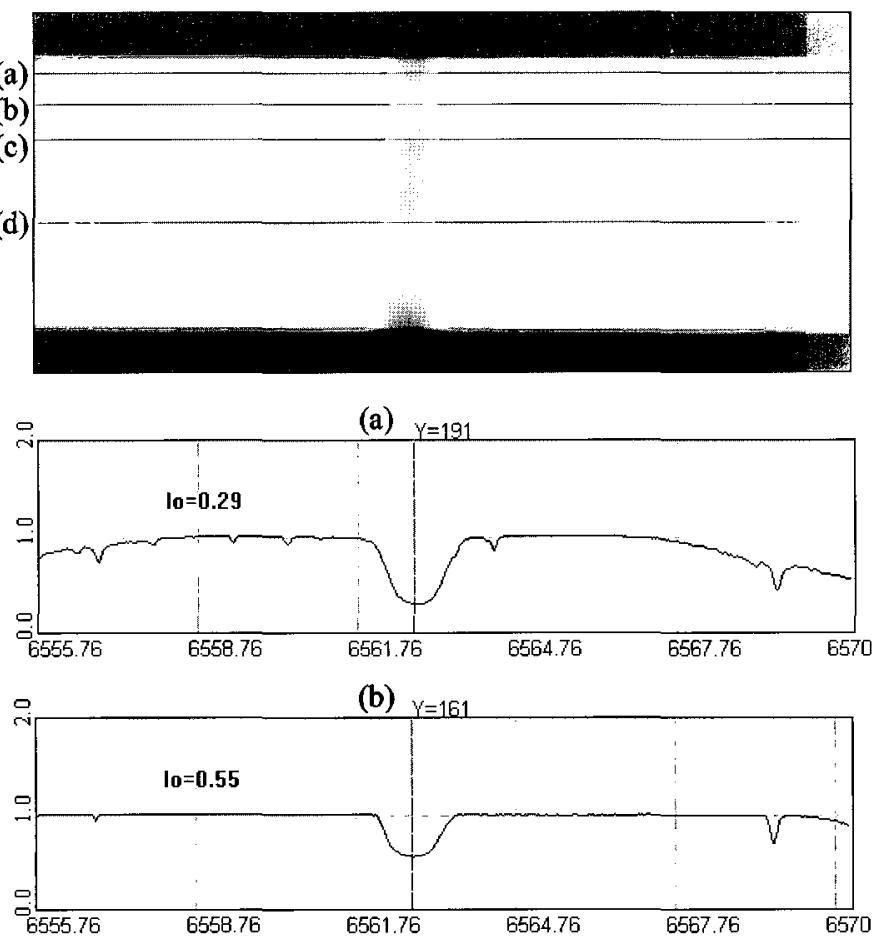

(c) $Y=143$
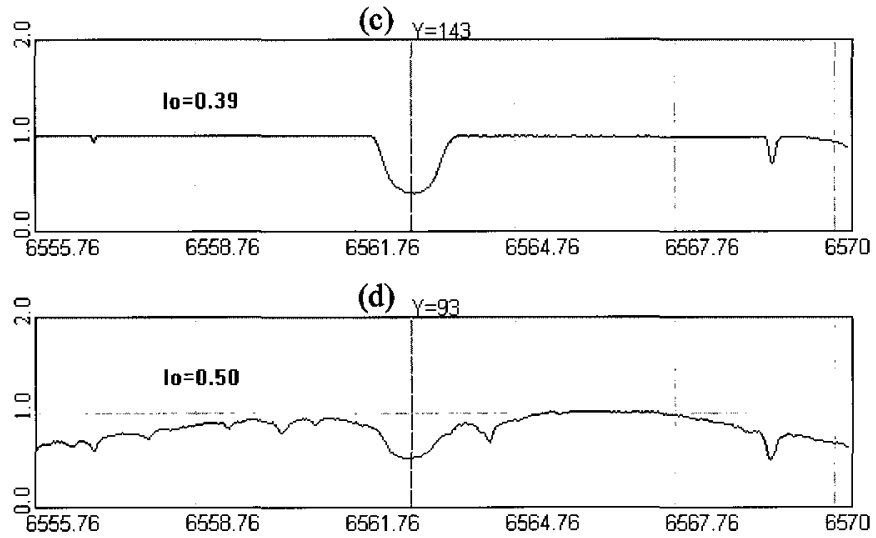

(e)

(f)

(g)

(h)

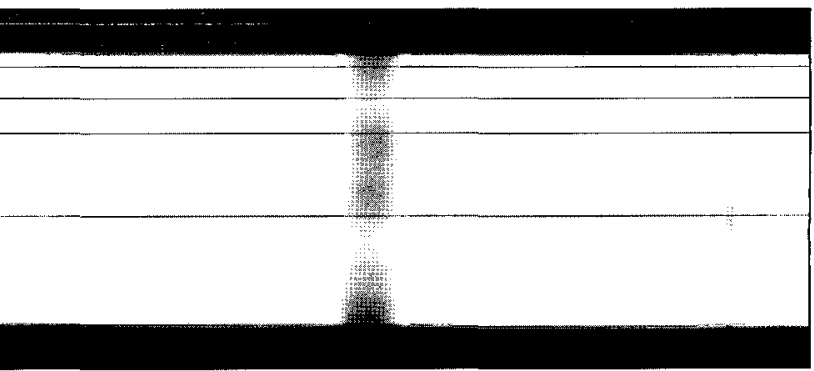

(e) $Y=184$
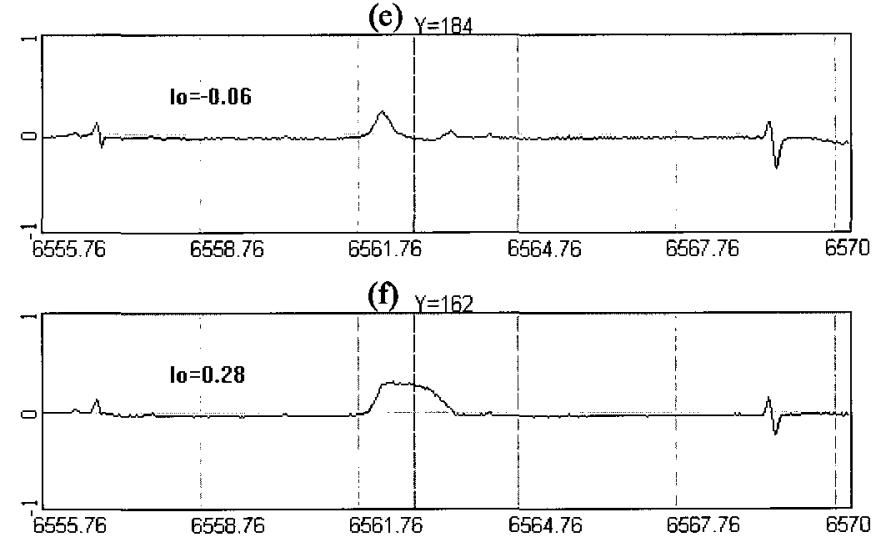

(g) $\gamma=144$

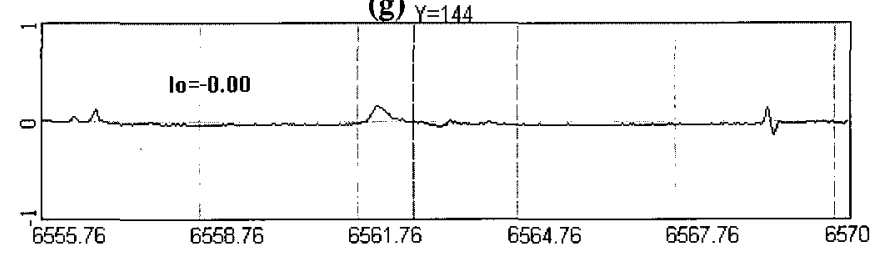

(h) $Y=94$

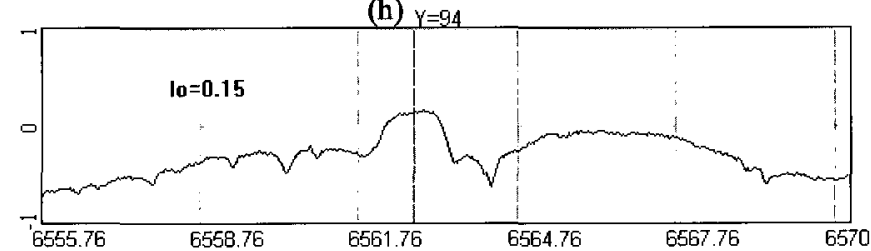

Fig. 10. - Spectrum profiles observed in the active region AR10708 

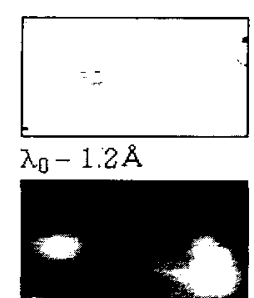

$\lambda_{0}-0.3 \AA$

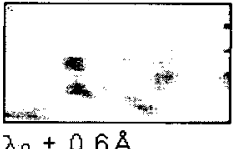

$\lambda_{0}+0.6 \AA$

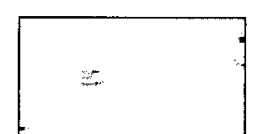

$\lambda_{0}-0.9 \AA$

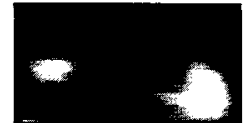

$\lambda_{0}=6562.81 \AA$

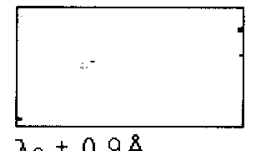

$\lambda_{0}+0.9 \AA$

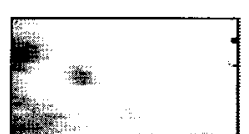

$\lambda_{0}-0.6 \AA$

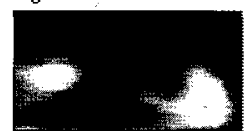

$\lambda_{0}+0.3 \AA$

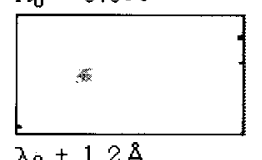

Fig. 11. - Spatial images of an active region AR 10708 for several selected wavelengths.

\section{SUMMARY AND DISCUSSION}

In this paper, we have made the component-based development of observational software for KSIS that is able to obtain three-dimensional imaging spectrograms by using a scanning mirror in front of the spectrograph slit. The observational software is developed by using component-based development methodology. The methodology gives us many benefits although it is difficult to understand and develop softwares based on it. Its main advantage is a very broad extension and reusability. We have adopted the COM (Component Object Model) technology for the observational software. And the software is developed via Microsoft Visual $\mathrm{C}++$ for Windows operating system. We expect that some components developed in this paper will be used for other observational softwares. The component-based development enables us to have the continuity of accumulated technical skills when developing new observational softwares. We think that a variety of data analysis will be prepared for better effective use of the KSIS.

Solar imaging spectrogram data have been obtained from the test observation of a sunspot (AR 10708). By using the developed software, we can clearly identified absorption and emission features near the $\mathrm{H} \alpha$ line center in the active region as well as continuum-like images at far wing regions. We think that the KSIS can be improved in the following aspects. First, it is necessary to stabilize the solar tracking system and the scanning system for better quality of the data. Second, it is possible to reduce observing time to take an imaging spectrogram with a faster CCD camera. Finally, we hope that the KSIS will play an important role in studying solar activity.

\section{ACKNOWLEDGEMENTS}

This work has been supported by the MOST funds (M1-0104-00-0059 and M1-0407-00-0001) of the Ko-

rean government. YJM and YDP were supported by the Korea Research Foundation (KRF-2005-070C00059) of the Korean Government. KSK thanks for the financial support of ABRL(R14-2002-043-01001-0). The NSO/Kitt Peak data used here were produced by NSF/NOAO.

\section{REFERENCES}

Bendlin, C., et al. 1992, A new instrument for high resolution, two-dimensional solar spectroscopy, A\&A, 257, 817

Canfield, R. C., et al. 1993, The Morphology of Flare Phenomena, Magnetic Fields, and Electric Currents in Active Regions. I. Introduction and Methods, ApJ, 411, 362

Ding, M. D., et al. 1995, Analysis of 2-D Flares Spectra : Velocity Fields Derived from Ha Line Asymmetries, SoPh, 158, 81

Geppatelli, C. \& Briand, C. 2003, THEMIS : Status and perspectives, Mem. S.A.It., 74, 790

Huang, Y. R., el al. 1995, A CCD imaging spectrograph in the improved solar tower of Nanjing University, SoPh, 159,127

Kurucz, R. L., Furenlid, I., Brault, James \& Testerman, Larry, 1984, Solar flux atlas from 296 to $1300 \mathrm{~nm}$, National Solar Observatory Atlas No. 1

Liu, Y. \& Ding, M. D. 2001, Physical Parameters of a Flare Derived from Multiline 2D Spectroscopy, ChJAA, 1, 460

Mein, P. 1991, Solar 2D spectroscopy : a new MSDP instrument, A\&A, 248, 669

Park, Y. D., et al. 2003, Development of Mid-resolution Solar Spectroscopic System, PKAS, 18, 61

Penn, M. J., et al. 1991, The Mees CCD imaging spectrograph, SoPh, 135, 163 